\title{
Teaching Large-Scale Digital Experimentation to Undergraduates and Graduate Students*
}

\author{
J. Nathan Matias \\ Princeton University ${ }^{\dagger}$
}

September 2018

\section{Introduction}

People with undergraduate degrees routinely conduct large-scale field experiments in online behavior, enabled by recent innovations in research software $[14,15,34]$. Since this is a recent development, relevant academic training remains primarily limited to graduate education, in classes that imagine small numbers of studies rather than the high-volume, software-guided experimentation common in industry. Because large-scale A/B testing is now common, universities have an opportunity to equip undergraduates and graduate students to design and carry out good experiments, make sense of results, and think critically about the societal implications of behavioral research at scale.

In this extended abstract, I review syllabi of classes about digital experimentation for their relevance to contemporary product and policy evaluation. I also describe SOC 412, a novel cross-disciplinary undergraduate and graduate class that I teach at Princeton University on the craft and ethics of digital experimentation at scale [20]. In the class, students learn to conduct multiple experiments and work in teams to design a large-scale field experiment online. The class is also a test environment for open educational resources for teaching online field experiments. I conclude the paper by identifying practical steps that academic educators and industry researchers can take to advance and spread undergraduate and graduate education in digital experimentation.

\section{The Experimenting Society}

Throughout the 20th century, social experiments in naturalistic settings provided rare, valuable methods to test pragmatic questions about the effects of policy and marketing interventions, sometimes also contributing to social theory $[24,7,25]$. At the time, the idea of an experimenting society - where many

\footnotetext{
*Pre-print and references are at https://osf.io/3pgrx/

$\dagger$ Department of Psychology and Center for Information Technology Policy
} 
thousands of experimenters continuously test small changes in products and institutions - seemed like a utopian thought experiment $[27,5]$. Now that digital information systems routinely monitor and intervene with billions of people in real-time, we live in that imagined society. Field experiments are essential to manage digital life, and society is just beginning to understand the implications.

While technology companies with hundreds of millions of users have led innovation in mass experimentation [15], this development is not limited to industry. Social scientists and policy evaluators are also using advances in data collection and collaboration infrastructures to scale the pace and reliability of research $[33,8]$. In civil society, field experiments are used for consumer protection research, auditing and evaluating the social impacts of technology products and policies $[28,16,1]$. Citizen behavioral science systems support the public to test behavioral products and evaluate citizen ideas for social change [22].

Transitions from bespoke research to mass experimentation involve changes in expertise and knowledge. Small teams who oversee every aspect of research are giving way to tool-makers who create research infrastructures that are used by others in ways that tool-makers may not anticipate [34]. The users of these tools include engineers, designers, copy-editors, and product managers who may never have been trained in social research, statistics, or research ethics. In some high-dimensional design areas, the entire process of experimentation has been automated, with algorithms developing intervention alternatives, conducting experiments, and making decisions with minimal ongoing human involvement [9]. In these settings, the average effect from one study can be less useful than heterogenous treatment effects [4] and data-mined meta-analyses.

The public mistrusts mass experimentation, which is largely secret. At such a high volume and distance from actual research, the designers of research systems may not always be able to anticipate the risks to participants. Furthermore, ethics procedures built around individual consent cannot match this pace of research, and experimenters have managed this mismatch by conducting most of their data collection and research in secret. Although field experiments might answer many fears about the social impacts of technology products, the public has responded to the secrecy of research with mistrust, concerned about manipulation from whoever holds experimenting power [29, 31].

How can democracies manage an experimenting society for the common good? This is one of the grand challenges of our time. Progress on this question requires a generation of researchers, engineers, statisticians, managers, advocates, and policymakers to engage closely with the details of research methods, technology design, policy, and ethics. Those pioneers will need training.

\section{Teaching Field Experiments}

In the spring of 2018, I taught SOC 412 [20], a hands-on undergraduate and graduate seminar class on field experiments in the Princeton department of Sociology and the certificate in Technology and Society. Students developed practical experimenting skills, engaging with methods, theory, ethics, and pol- 
itics of large-scale behavioral research online [21]. For a final project, student teams developed a novel experiment together with an online community.

\section{Experiment Class Syllabi}

I created this class because most training in experiments, even at the graduate level, focuses on research design and analysis, inviting students to read, estimate, and comment on published research $[6,26,32,30,12,2,11]$. Only two syllabi I reviewed support students to design and conduct their own experiments $[23,10]$. All of the class syllabi were designed for small-scale, bespoke experimentation by scientists in political science, economics, and business.

\section{SOC412: Designing Field Experiments at Scale}

The audience for SOC412 are students from policy, social science, and engineering who have introductory statistics knowledge, and who wish to grow as experimenters and to think critically about the societal implications of large-scale behavioral research. In the class, teams of students design a novel digital field experiment, think about that study from the perspective of mass experimentation, and write personal essays about the social and ethical issues. Students co-developed many of their projects together with large communities on the reddit platform. In the first year of this class, students developed projects to:

- test interventions to improve people's resilience against organized attempts to demoralize and push people away from an online community

- evaluate ideas to prevent unruly behavior in online communities through nudges that monitor news aggregators that cause large-scale conflict

- test automated systems that could help people ask better questions in large online discussions

- crowd-source n-of-one trials of common theories about phone addiction.

- audit social media platform policies

Each student also developed a final paper that discussed an ethical or political question raised by their research project. For example, students wrote on the epistemology and policy of replications, the role of measurement in generalizable knowledge, the ethics of risky experiments involving trauma, methods for crowdsourcing experiment ideas from a wider public, difficulties of estimating risk/benefits in conflict situations, and legal risks of auditing social platforms.

\section{Resources for Teaching Digital Experimentation}

To support SOC 412, I developed a series of resources, class units, and strategies teaching field experiments. ${ }^{1}$

\footnotetext{
${ }^{1}$ Lectures and code are available online: https://github.com/natematias/SOC412
} 


\section{The Social Media Color Experiment}

The introductory sequence of units in SOC412 supports students to conduct an experiment called the "social media color experiment" [17, 19] In this experiment coordinated through a spreadsheet, students are supported to randomly assign a single social media post per day to have a colored or white background. They then record the number of interactions that the post receives and estimate the results. Because every student conducts their own study, this simple experiment offers students their first encounters with the basics of experimentation, replication, family-wise error rates, and meta-analysis over several weeks. Since participants were students' own friends and family members, students could consider risks and benefits for people they knew when writing about ethics.

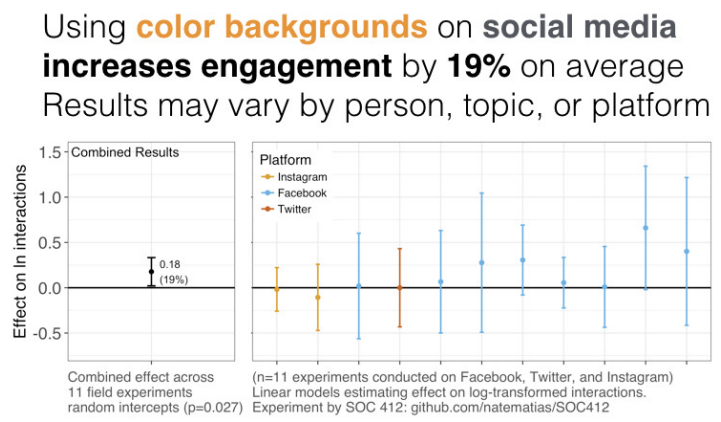

\section{Teaching with Experimentation Infrastructures}

In SOC412, several final projects made use of the CivilServant experimentation system [22]. The system conducts data collection, managing interventions, and managing ethics procedures for industry-independent research on the reddit platform. Since CivilServant carried out the work of experimentation, students were able to focus on the design of their study while learning how experimentation infrastructures work. Furthermore, since the system was already collecting continuous behavioral data where communities granted consent, students were able to use prior data to develop study designs and estimate statistical power.

\section{Ethics Board Review for Class Experiments}

For a class on field experiments to succeed, the university's ethics board (IRB) needs to work on the timeline of a single semester. To streamline this process, I notified Princeton's IRB about the class in advance and filed rough drafts of anticipated experiment plans for approval. I then amended the ethics protocols partway through the semester, adding the names of students who took up a project and adjusting protocols to follow student research decisions. 


\section{Future Educational Resources and Opportunities}

Just as software has transformed the work of experimenting, software and data could support classes like SOC412 to scale and become more common [18]. Toward that goal, I have identified several areas where academic educators, industry, and civil society can work together to further develop education on the craft, ethics, and societal implications of large-scale experimentation.

- Datasets of historical experiments. Companies, news organizations, and advocacy organizations currently hold large datasets with the results of thousands of field experiments and the organization's accompanying decisions. If organizations publish anonymized observations from large sets of experiments that are no longer relevant to their operations, students could use that data to gain practical experience in validating and analyzing results from mass experimentation.

- Case studies on experimentation. The only available case study on large-scale behavioral research is the Facebook Emotion Contagion study, which is useful but insufficient [13]. On issues of AI and ethics, corporations, governments, and educators have co-authored case studies on decisions and trade-offs in real-world situations [35]. Similar case studies on experimentation could contribute substantially to university education.

- Open experiment design resources. Scientific researchers have recently begun to create open resources that express the full details of experiment design and analysis in software [3]. This software simplifies the process of power analysis and estimation. Modules designed for online behavioral measurements would simplify research design for students.

\section{Conclusion}

Digital experimentation is changing the nature of knowledge and power in the 21st century. If policymakers, companies, and civil society are to manage this transition well, we need capable and innovative people who can contribute to an experimenting society. Universities can train those leaders - if students are supported with classes that provide students with solid introductions to the technical, methodological, and societal dimensions of experimentation at scale.

\section{Acknowledgments}

I am grateful to Martin Saveski for an archive of syllabi that provided the seed for my class research and the syllabi cited in this abstract. 


\section{References}

[1] Susan Benesch and J. Nathan Matias. Launching today: new collaborative study to diminish abuse on Twitter, April 2018. URL https://medium.c om/@susanbenesch/launching-today-new-collaborative-study-to-d iminish-abuse-on-twitter-2b91837668cc.

[2] Matthew Blackwell. Gov 2002: Causal Inference. Undergraduate, Harvard Kennedy School, 2015.

[3] Graeme Blair, Jasper Cooper, Alexander Coppock, and Macartan Humphreys. Declare-Design Version 1.0. Software package for R, available at http://declaredesign. org, 2016.

[4] Netflix Technology Blog. Artwork Personalization at Netflix, December 2017. URL https://medium.com/netflix-techblog/artwork-personal ization-c589f074ad76.

[5] Donald T. Campbell. Reforms as experiments. American Psychologist, 24(4):409-429, 1969. ISSN 1935-990X(Electronic);0003-066X(Print). doi: $10.1037 / \mathrm{h} 0027982$.

[6] Carolina Castilla. Field Experiments in a Course on Behavioral Economics: Nudging Students Around Campus. The Journal of Economic Education, 45(3):211-224, July 2014. ISSN 0022-0485. doi: 10.1080/00220485.2014.91 7566. URL https://doi.org/10.1080/00220485.2014.917566.

[7] Robert B. Cialdini. Full-cycle social psychology. Applied social psychology annual, 1980. URL http://psycnet.apa.org/psycinfo/1981-23592-00 1.

[8] Open Science Collaboration and others. An open, large-scale, collaborative effort to estimate the reproducibility of psychological science. Perspectives on Psychological Science, 2012. URL http://journals.sagepub.com/d oi/abs/10.1177/1745691612462588.

[9] Vito Covalucci. AI as complement to A/B test design, September 2017. URL https://hackernoon.com/ai-as-complement-to-a-b-test-desi gn-e8f $4 b 5 e 28 d 92$.

[10] Esther Duflo. Putting Social Sciences to the Test: Field Experiments in Economics. Undergraduate Class, MIT Economics, 2006. URL https:// ocw.mit.edu/courses/economics/14-11-putting-social-sciencesto-the-test-field-experiments-in-economics-spring-2006/.

[11] Dean Eckles. Experimental Design. PhD Seminar, MIT Sloan Business School, 2016.

[12] Alan Gerber and Donald Green. Experimental Methods in Political Science. Technical report, Yale University Political Science, 2010. 
[13] James Grimmelmann. The law and ethics of experiments on social media users. J. on Telecomm. \& High Tech. L., 13:219, 2015.

[14] Katja Kevic, Brendan Murphy, Laurie Williams, and Jennifer Beckmann. Characterizing experimentation in continuous deployment: a case study on bing. In Proceedings of the 39th International Conference on Software Engineering: Software Engineering in Practice Track, pages 123-132. IEEE Press, 2017. URL http://dl.acm.org/citation. cfm?id=3103129.

[15] Ron Kohavi, Alex Deng, Brian Frasca, Toby Walker, Ya Xu, and Nils Pohlmann. Online Controlled Experiments at Large Scale. In Proceedings of the 19th ACM SIGKDD International Conference on Knowledge Discovery and Data Mining, KDD '13, pages 1168-1176, New York, NY, USA, 2013. ACM. ISBN 978-1-4503-2174-7. doi: 10.1145/2487575.2488217. URL http: //doi.acm.org/10.1145/2487575.2488217.

[16] J. Nathan Matias. The Obligation To Experiment, December 2016. URL https://medium.com/mit-media-lab/the-obligation-to-exper iment-83092256c3e9.

[17] J. Nathan Matias. How Anyone Can Audit Facebook's NewsFeed, December 2017. URL https://medium.com/@natematias/how-anyone-can-a udit-facebooks-newsfeed-b879c3e29015.

[18] J. Nathan Matias. Can Classes on Field Experiments Scale? Lessons from SOC412, July 2018. URL https://freedom-to-tinker.com/2018/07/16 /can-classes-on-field-experiments-scale-lessons-from-soc412/.

[19] J. Nathan Matias. Is It Just Me? Pooling Experiments to Audit Common Impacts of Social Tech, May 2018. URL https://medium. com/@natematia s/is-it-just-me-pooling-experiments-to-audit-common-impactsof-social-tech-ca9fdb78516.

[20] J. Nathan Matias. SOC 412: Designing Field Experiments at Scale, January 2018. URL https://natematias.com/courses/soc412/syllabus.h tml.

[21] J. Nathan Matias. Teaching the Craft, Ethics, and Politics of Field Experiments, July 2018. URL https://freedom-to-tinker.com/2018/07/03/t eaching-the-craft-ethics-and-politics-of-field-experiments/.

[22] J. Nathan Matias and Merry Mou. CivilServant: Community-Led Experiments in Platform Governance. In Proceedings of the 2018 CHI Conference on Human Factors in Computing Systems, page 9. ACM, 2018.

[23] Brendan Nyhan. GOVT 83.21 / QSS 30.03 Experiments in Politics. Undergraduate Class, Dartmouth University Political Science. URL http: //www.dartmouth. edu/ nyhan/experiments-syllabus.pdf. 
[24] Ann Oakley. Experimentation and social interventions: a forgotten but important history. BMJ: British Medical Journal, 317(7167):1239, 1998. URL https://www.ncbi.nlm.nih.gov/pmc/articles/PMC1114168/.

[25] Elizabeth Levy Paluck and Robert B. Cialdini. Field research methods. Handbook of research methods in social and personality psychology, pages 81-97, 2014. URL https://static1.squarespace.com/static/5186d 08fe4b065e39b45b91e/t/55da52f0e4b0838dce537392/1440371440284 /Paluck_Cialdini_2014.pdf.

[26] Rosalind Picard. MAS S.64 Tools for Causal Inference. Graduate Course, MIT Media Lab, 2018.

[27] Karl Popper. The open society and its enemies. Routledge, 1947.

[28] Christian Sandvig, Kevin Hamilton, Karrie Karahalios, and Cedric Langbort. Auditing algorithms: Research methods for detecting discrimination on internet platforms. Data and discrimination: converting critical concerns into productive inquiry, 2014. URL https://pdfs. semanticschol ar.org/b722/7cbd34766655dea10d0437ab10df3a127396.pdf.

[29] Noam Scheiber. How Uber Uses Psychological Tricks to Push Its Drivers' Buttons. The New York Times, April 2017. ISSN 0362-4331. URL https://www.nytimes.com/interactive/2017/04/02/technolog y/uber-drivers-psychological-tricks.html.

[30] Jasjeet Singh Sekhon. The Statistics of Causal Inference in the Social Sciences. Undergraduate, Berkeley Political Science.

[31] Aaron Smith. How Americans View Tech Companies. Technical report, Pew Research Center, June 2018. URL http://www . pewinternet. org/20 18/06/28/public-attitudes-toward-technology-companies/.

[32] Johan Ugander. MS\&E334: The Structure of Social Data. Graduate Course, Stanford University Management Science \& Engineering, 2016.

[33] Eric Luis Uhlmann, Christopher R. Chartier, Charles R. Ebersole, Tim Errington, Mallory C. Kidwell, Calvin Lai, Randy J. McCarthy, Amy Riegelman, Raphael Silberzahn, and Brian A. Nosek. Scientific Utopia: III. Crowdsourcing Science. 2018. URL https://psyarxiv.com/vg649.

[34] Ya Xu, Nanyu Chen, Addrian Fernandez, Omar Sinno, and Anmol Bhasin. From infrastructure to culture: A/b testing challenges in large scale social networks. In Proceedings of the 21th ACM SIGKDD International Conference on Knowledge Discovery and Data Mining, pages 2227-2236. ACM, 2015. URL http://dl . acm. org/citation. cfm?id=2788602.

[35] Bendert Zevenbergen, Chloé Bakalar, Melissa Lane, and Edward Felten. Case Studies: Dialogue on AI and Ethics. Technical report, Princeton University, April 2018. URL https://aiethics.princeton.edu/casestudies/. 